\title{
Long Rotational Coherence Times of Molecules in a Magnetic Trap
}

\author{
L. Caldwellø, ${ }^{1}$ H. J. Williams $\odot,{ }^{1}$ N. J. Fitch $\odot,{ }^{1}$ J. Aldegunde $\odot,{ }^{2}$ Jeremy M. Hutson $\odot,{ }^{3}$ \\ B. E. Sauer $\odot,{ }^{1}$ and M. R. Tarbutt $\odot^{1}$ \\ ${ }^{1}$ Centre for Cold Matter, Blackett Laboratory, Imperial College London, \\ Prince Consort Road, London SW7 2AZ, United Kingdom \\ ${ }^{2}$ Departamento de Quimica Fisica, Universidad de Salamanca, E-37008 Salamanca, Spain \\ ${ }^{3}$ Joint Quantum Centre (JQC) Durham-Newcastle, Department of Chemistry, Durham University, \\ South Road, Durham DH1 3LE, United Kingdom
}

(Received 30 August 2019; accepted 17 December 2019; published 10 February 2020)

\begin{abstract}
Polar molecules in superpositions of rotational states exhibit long-range dipolar interactions, but maintaining their coherence in a trapped sample is a challenge. We present calculations that show many laser-coolable molecules have convenient rotational transitions that are exceptionally insensitive to magnetic fields. We verify this experimentally for $\mathrm{CaF}$ where we find a transition with sensitivity below $5 \mathrm{~Hz} \mathrm{G}^{-1}$ and use it to demonstrate a rotational coherence time of 6.4(8) $\mathrm{ms}$ in a magnetic trap. Simulations suggest it is feasible to extend this to more than $1 \mathrm{~s}$ using a smaller cloud in a biased magnetic trap.
\end{abstract}

DOI: 10.1103/PhysRevLett.124.063001

Ultracold polar molecules present exciting opportunities for quantum simulation, quantum computation, and tests of fundamental physics [1]. The rotational motion is particularly important for these applications. Rotational transitions are easily driven by microwave fields, and excited rotational states have long lifetimes. Polar molecules in superpositions of rotational states have large oscillating electric dipole moments, providing long-range dipole-dipole interactions between them [2-4]. The strong electric dipole coupling of rotational states to microwave fields can also be used to interface gas-phase molecules with mesoscopic solid-state systems [5,6]. Many schemes have been proposed to use these interactions to generate entanglement, engineer interparticle potentials, and implement two-qubit quantum gates [7-11].

Most applications require trapped molecules and coherence times that are long compared to the characteristic interaction strength. Here, we explore how that can be achieved with molecules in magnetic traps. To avoid the dephasing that arises from an inhomogeneous transition frequency, the trap potential should, ideally, be identical for the two rotational states. Experiments so far have focused exclusively on optical traps in the form of optical lattices or arrays of tweezer traps where interparticle separations below $1 \mu \mathrm{m}$ are possible, producing dipole-dipole couplings with energies $\sim 1 \mathrm{kHz}$. While second-long coherence

Published by the American Physical Society under the terms of the Creative Commons Attribution 4.0 International license. Further distribution of this work must maintain attribution to the author(s) and the published article's title, journal citation, and DOI. times have been demonstrated for superpositions of hyperfine states [12], rotational coherence times longer than $\sim 1 \mathrm{~ms}$ are a challenge because of the dependence of the ac Stark shift on the rotational state [13]. By using a dc electric field to uncouple the rotational angular momentum from the nuclear spin, and setting the polarization angle of the trapping light to equalize the Stark shifts of the chosen rotational states, Seeßelberg et al. [14] extended rotational coherence times to 8.7(6) $\mathrm{ms}$ in a sample of optically trapped $\mathrm{NaK}$ molecules. Long vibrational coherence times have also been demonstrated for $\mathrm{Sr}_{2}$ molecules in a stateinsensitive optical lattice [15].

Recently, direct laser cooling has produced ultracold molecules with both electric and magnetic moments [16-19], opening new possibilities for experiments in magnetic traps [20,21]. Two-dimensional arrays of magnetic traps have been demonstrated for atoms with spacings comparable to those achievable with optical arrays [22]. Molecules might also be held in magnetic chip traps close to superconducting microwave resonators [5], where the regime of strong coupling between molecules and microwave photons may be reached, and where molecules may be coupled via the resonator with interaction strengths of order $100 \mathrm{kHz}$.

To obtain long rotational coherence times in a magnetic trap, it is necessary to find states with large and nearly identical magnetic moments. Here, we investigate rotational transitions in ${ }^{2} \Sigma$ molecules and find a pair of states in $\mathrm{CaF}$ with magnetic moments of $\sim 1 \mu_{B}$ that are equal to 3.3(1) parts per million. We demonstrate a superposition of the two states in a quadrupole magnetic trap with a coherence time of 6.4(8) ms. By comparison with simulations and freespace measurements we establish the dominant dephasing 
mechanisms and propose ways to achieve coherence times exceeding $1 \mathrm{~s}$.

The interaction of a ${ }^{2} \Sigma$ molecule with a magnetic field $\vec{B}$ is described by the Zeeman Hamiltonian [23,24],

$$
\begin{aligned}
H_{Z}= & H_{e}+H_{n}+H_{r}+H_{a} \\
= & g_{S} \mu_{B} \vec{S} \cdot \vec{B}-\sum_{i} g_{N}^{i} \mu_{N} \vec{I}^{i} \cdot \vec{B}-g_{r} \mu_{B} \vec{N} \cdot \vec{B} \\
& +g_{l} \mu_{B}[\vec{S} \cdot \vec{B}-(\vec{S} \cdot \hat{z})(\vec{B} \cdot \hat{z})],
\end{aligned}
$$

where $\hat{z}$ is a unit vector in the direction of the internuclear axis, $\vec{S}$ is the electron spin operator, $\vec{N}$ is the rotational angular momentum operator, $\vec{I}^{i}$ is the spin operator of nucleus $i$, and the sum is over the nuclei. $H_{e}$ describes the interaction of the electron magnetic moment with $\vec{B}$ and is, by far, the largest term. $H_{n}$ describes the much smaller contribution from the nuclear magnetic moments, $H_{r}$ the rotational Zeeman interaction, and $H_{a}$ the anisotropic correction to the electronic Zeeman interaction. The search for magnetically insensitive rotational transitions is hindered by the Hamiltonian for the fine and hyperfine structure, $H_{\text {fhf }}$, which couples the angular momenta in a way that depends on $N$ [23]. This is solved by choosing the stretched states $|N\rangle_{\text {str }}=\left|N, m_{N}=N\right\rangle\left|S, m_{S}=S\right\rangle\left|I, m_{I}=I\right\rangle$, where $m_{X}$ is the projection of $X$ onto the magnetic field axis. These states are eigenstates of $H_{\text {fhf }}$ and $H_{Z}$. Their Zeeman shifts, $\Delta E_{N}$, are almost identical, because the large contribution from $H_{e}$, and the smaller one from $H_{n}$, are both independent of $N$. For the single-photon transitions $|N\rangle_{\text {str }} \leftrightarrow|N+1\rangle_{\text {str }}$, the residual magnetic sensitivity due to the two remaining terms is

$$
\begin{aligned}
\Delta \mu(N) & =\left(\Delta E_{N+1}-\Delta E_{N}\right) / B, \\
& =\left(\frac{g_{l}}{(2 N+4)^{2}-1}-g_{r}\right) \mu_{B} .
\end{aligned}
$$

If the ratio $g_{l} / g_{\mathrm{r}}$ is close to $(2 N+4)^{2}-1$ for some $N$, the remaining two terms nearly cancel, giving the desired magnetic insensitivity.

We have carried out calculations of this ratio for a variety of alkaline-earth fluorides, hydrides, and hydroxides of interest for laser cooling. To evaluate $g_{l}=\Delta g_{\perp}=g_{\|}-g_{\perp}$, we calculate the parallel and perpendicular components of the molecular $g$ tensor by density-functional theory (DFT) as implemented in ORCA [25], using the B3LYP functional [26] and x2c-TZVPP all-electron basis sets [27]. Relativistic corrections are included by the zero-order regular approximation (ZORA) [28-30], but are small for the molecules considered here. We evaluate $g_{r}$ from Hartree-Fock calculations, as implemented in DALTON [31], using the same basis sets. Further details are given in the Supplemental Material [32].

The results obtained when the bond length for each molecule is fixed at its equilibrium value $R_{e}$ are summarized in Table I. Figure 1 shows the resulting $\Delta \mu$ for transitions $|N\rangle_{\text {str }} \leftrightarrow|N+1\rangle_{\text {str }}$ up to $N=6$. For $N=0$, the sensitivities are dominated by $g_{l}$, and for large $N$ they approach the value set by $g_{r}$. The hydrides generally have much larger values of both $g_{r}$ and $g_{l}$ and have no transitions with residual sensitivities on the scale shown. The remaining molecules each exhibit at least one transition with $|\Delta \mu / h|<30 \mathrm{~Hz} \mathrm{G}^{-1}$ and six of them have a transition with $|\Delta \mu / h|<10 \mathrm{~Hz} \mathrm{G}^{-1}$. These results suggest that convenient rotational transitions with extremely small magnetic sensitivities are a common feature of alkaline-earth fluorides and hydroxides.

We have used Ramsey spectroscopy in a uniform magnetic field $B$ to determine the magnetic sensitivities of such transitions experimentally in $\mathrm{CaF}$. We measure $\Delta \mu$ for two transitions, $|0\rangle_{\text {str }} \leftrightarrow|1\rangle_{\text {str }}$ and $|1\rangle_{\text {str }} \leftrightarrow|2\rangle_{\text {str }}$, whose frequencies are $f_{0 \leftrightarrow 1}=20553.4$ and $f_{1 \leftrightarrow 2}=41088.9 \mathrm{MHz}$. We capture $\sim 10^{4}$ molecules in a magneto-optical trap [17], cool them to about $50 \mu \mathrm{K}$ [48], then prepare them in a single internal state, either $|0\rangle_{\text {str }}$ or $|1\rangle_{\text {str }}$ [21]. The Ramsey sequence [49] uses a pair of nearly resonant $\pi / 2$ pulses, $20 \mu$ s long, separated by a free-evolution time $\tau$. Following the second pulse, we measure the number of molecules in $N=1$ by recapturing them into the MOT and imaging their fluorescence. Dividing by the number initially loaded in the MOT gives a signal that is insensitive to shot-to-shot fluctuations in the number of molecules. The magnetic field at the molecules is calibrated to better than $1 \%$ by single-pulse microwave spectroscopy of the transition $\left|N=0, F=1, m_{F}=-1\right\rangle \leftrightarrow$ $|N=1, F=0\rangle$. The Zeeman shift of this transition can be accurately calculated because it is dominated by $H_{e}$, and because $g_{S}$ and the hyperfine parameters are known to high precision [50].

TABLE I. Results of electronic structure calculations for $g_{l}$ and $g_{r}$ for a variety of potentially laser-coolable molecules at their equilibrium bond lengths $R_{e}$.

\begin{tabular}{lcccccccccccr}
\hline \hline Molecule & $\mathrm{BeF}$ & $\mathrm{MgF}$ & $\mathrm{CaF}$ & $\mathrm{SrF}$ & $\mathrm{BeH}$ & $\mathrm{MgH}$ & $\mathrm{CaH}$ & $\mathrm{SrH}$ & $\mathrm{BeOH}$ & $\mathrm{MgOH}$ & $\mathrm{CaOH}$ & $\mathrm{SrOH}$ \\
\hline$R_{e}(\mathrm{M}-\mathrm{X})(\AA)$ & 1.36 & 1.75 & 1.95 & 2.07 & 1.34 & 1.73 & 2.00 & 2.15 & 1.37 & 1.76 & 2.03 & 2.16 \\
$R_{e}(\mathrm{O}-\mathrm{H})(\AA)$ & $\ldots$ & $\ldots$ & $\ldots$ & $\ldots$ & $\ldots$ & $\ldots$ & $\ldots$ & $\ldots$ & 0.94 & 0.94 & 0.95 & 0.97 \\
$10^{3} g_{l}$ & -0.820 & -1.74 & -1.86 & -4.97 & -0.111 & -2.18 & -4.20 & -15.1 & -0.394 & -1.26 & -1.73 & -5.24 \\
$10^{5} g_{r}$ & -7.36 & -3.73 & -5.13 & -4.77 & -144 & -88.5 & -106 & -111 & -2.18 & -1.48 & -3.31 & -3.17 \\
$g_{l} / g_{r}$ & 11.1 & 46.6 & 36.3 & 104 & 0.077 & 2.46 & 3.95 & 13.6 & 18.0 & 85.0 & 52.3 & 165 \\
\hline \hline
\end{tabular}




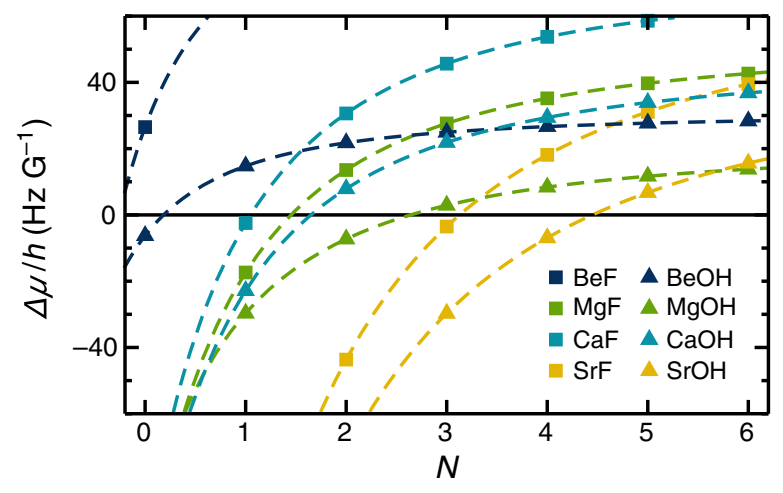

FIG. 1. Calculated magnetic sensitivities of transitions $|N\rangle_{\text {str }} \leftrightarrow$ $|N+1\rangle_{\text {str }}$ for a variety of alkaline-earth fluorides and hydroxides of interest for laser cooling.

Figure 2(a) shows Ramsey fringes for the transition $|1\rangle_{\text {str }} \leftrightarrow|2\rangle_{\text {str }}$, obtained by scanning the microwave frequency. The dark blue data are for $\tau=4 \mathrm{~ms}$ and the light blue for $\tau=5 \mathrm{~ms}$. Repeating the measurement with different $\tau$ identifies the fringe corresponding to the center frequency. Fitting to the fringes determines the transition frequency with statistical uncertainty below $1 \mathrm{~Hz}$.

Figure 2(b) shows the change in transition frequency for both transitions as a function of $B$. The solid lines show linear fits to the data that give residual magnetic sensitivities $\Delta \mu / h=-104(4) \mathrm{Hz} \mathrm{G}^{-1}$ for the transition

(a)

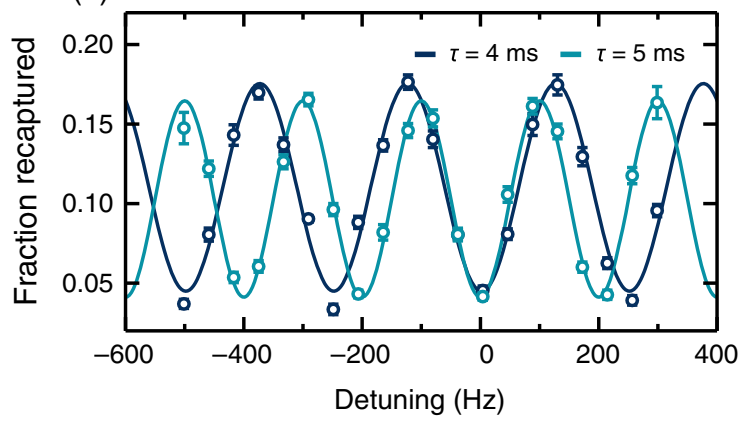

(b)

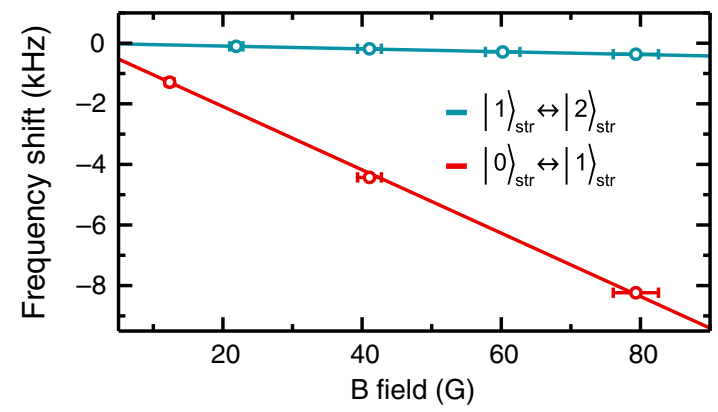

FIG. 2. (a) Ramsey spectroscopy of the transition $|1\rangle_{\text {str }} \leftrightarrow|2\rangle_{\text {str }}$ in free space with $\tau=4$ and $5 \mathrm{~ms}$. Points and error bars: mean and standard error of 19 repeated experiments; lines: sinusoidal fits to the data. (b) Magnetic sensitivity of the transitions $|0\rangle_{\text {str }} \leftrightarrow|1\rangle_{\text {str }}$ (red) and $|1\rangle_{\text {str }} \leftrightarrow|2\rangle_{\text {str }}$ (light blue). Lines: linear fits to the data. Vertical error bars are smaller than the points. $|0\rangle_{\text {str }} \leftrightarrow|1\rangle_{\text {str }}$ and $-4.7(2) \mathrm{HzG}^{-1}$ for $|1\rangle_{\text {str }} \leftrightarrow|2\rangle_{\text {str }}$ The uncertainties are dominated by drifts in $B$ between measurements of the insensitive transition and the transition used for calibration, represented by the horizontal error bars. The error in magnetic field is correlated between different points, because the drift is slow compared to the measurement time; this has been taken into account in the calculation of the uncertainties in $\Delta \mu$. From these measurements we determine $g_{l}=-1.87(8) \times 10^{-3}$ and $g_{r}=-5.0(2) \times 10^{-5}$, in excellent agreement with the calculated values. At $N=1$ the two terms in Eq. (2) cancel to within $3 \%$, resulting in cancellation of the magnetic moments of the two states to 3.3(1) parts per million.

The magnetic insensitivity of the transition $|1\rangle_{\text {str }} \leftrightarrow|2\rangle_{\text {str }}$ suggests that long coherence times should be possible in a magnetic trap. To investigate this we measure the decay of the beat note between the oscillations of the rotational superposition and those of a slightly detuned microwave field. The first $\pi / 2$ pulse is applied and then the quadrupole trap is immediately turned on with an axial field gradient of $45 \mathrm{G} \mathrm{cm}^{-1}$. The lifetime of the molecules in the magnetic trap is about $4.5 \mathrm{~s}$ [21], much longer than the timescale of any of the experiments reported here. The second $\pi / 2$ pulse is applied in the trap and the molecules are then recaptured and imaged in a MOT, as before. Figure 3(a) shows the Ramsey fringes traced out by scanning the free evolution time $\tau$. The solid line is a fit to a sinusoid with exponentially decaying amplitude, which gives a coherence time of 6.4(8) $\mathrm{ms}$.

To elucidate the sources of decoherence in the trap, we use a Monte Carlo simulation of the Ramsey experiment in the magnetic trap. The result of this simulation fits well to an exponentially decaying sinusoid with $1 / e$ time of $6.1 \mathrm{~ms}$, in agreement with our experimental data. We identify three decoherence mechanisms: (i) The residual magnetic sensitivity of the transition causes a spread in the phase accumulated by different molecules. (ii) The movement of a molecule between the two $\pi / 2$ pulses causes a change of phase which is different for each molecule; this is a form of Doppler broadening. (iii) The molecules adiabatically follow the local magnetic field vector as they move along different trajectories. This last mechanism has two effects. The first is to vary the amplitude of the microwave field in the relevant polarisation for each molecule at each Ramsey pulse, resulting in a loss of contrast, but no longer-term dephasing. The second is to impart a geometric phase to each molecule, which depends on its trajectory between the two pulses, and is proportional to the difference in $m_{F}$ between the two states [51]. To quantify the contributions of each mechanism, we artificially remove the effect of the others from the simulation and find the time taken for the amplitude of the Ramsey fringes to decay by a factor of $e$. We find the decoherence rates for each mechanism to be (i) $60 \mathrm{~s}^{-1}$, (ii) $50 \mathrm{~s}^{-1}$, and (iii) $100 \mathrm{~s}^{-1}$. 

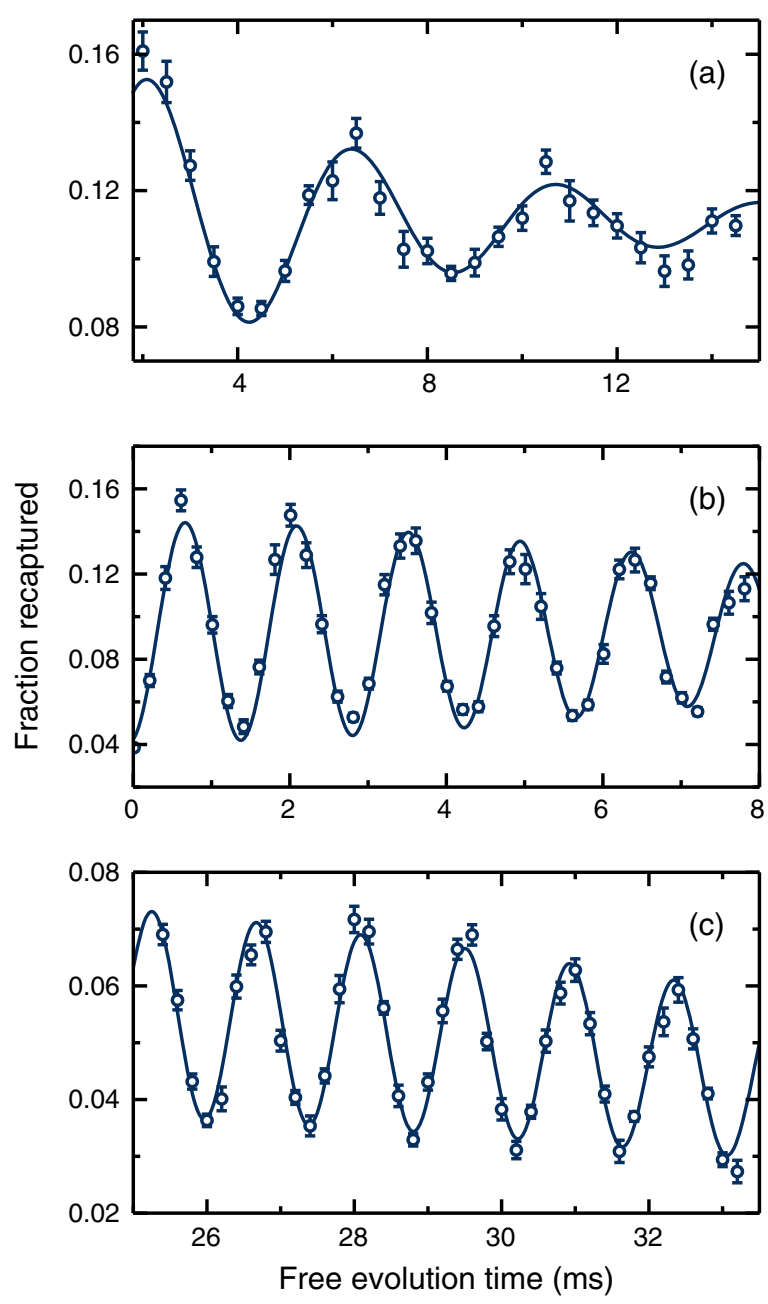

FIG. 3. (a) Ramsey fringes for molecules in the magnetic trap. Line: fit to $A+B e^{-\alpha t} \cos (\Delta t+\phi)$ giving a coherence time $1 / \alpha=6.4(8) \mathrm{ms}$. (b) Ramsey fringes for molecules in free space. Line: fit to $A+B e^{-\alpha^{2} t^{2}} \cos (\Delta t+\phi)$, with $1 / \alpha=(\lambda / 2 \pi) \times$ $\sqrt{m / 2 k_{B} T}=11.4(6) \mathrm{ms}$, corresponding to $T=37(4) \mu \mathrm{K}$. (c) As (b) but with a spin echo applied: an extra $\pi$ pulse is applied, $14.7 \mathrm{~ms}$ after the first $\pi / 2$ pulse. The $x$ axis shows total freeevolution time between first and second $\pi / 2$ pulses. Line: fit to $\left(1-C t^{2}\right)\left[A+B e^{-\alpha t} \cos (\Delta t+\phi)\right]$. The loss of fringe contrast is consistent with $\alpha=0$. Points and error bars throughout show mean and standard error of 19 repeated experiments.

The decoherence rate of mechanism (i) scales linearly with the average field experienced by the molecules in the trap, currently set almost entirely by the initial Gaussian width of $1.5 \mathrm{~mm}$. In the limit of a small initial cloud, the sample explores a range of fields determined only by its temperature $T$. The resulting spread of transition frequencies, $\left(k_{B} T / h\right)\left(\Delta \mu / \mu_{B}\right)$, gives a decoherence rate of $0.14 \mu \mathrm{K}^{-1} \mathrm{~s}^{-1}$. This implies an achievable coherence time of $1.4 \mathrm{~s}$ at $5 \mu \mathrm{K}$, a temperature that has already been demonstrated for $\mathrm{CaF}[52,53]$. The effect of mechanism (ii) is also related to the cloud size. When the maximum distance that a molecule moves is less than the wavelength of the microwave field, the phase change due to motion is limited to less than $2 \pi$ and the superposition never fully decoheres. The result is a fixed loss of contrast at late times. In our experiment, this maximum phase change is set by the initial cloud size; reducing the cloud size would decrease the size of the effect. Mechanism (iii), currently the largest contribution to the decoherence rate, can be greatly reduced by using a magnetic trap geometry with a large bias field [54] to ensure that there is little variation of the magnetic field direction in the region where the molecules are confined.

To identify any decoherence mechanisms unrelated to the trap, we repeated the decoherence measurement in free space, as shown in Fig. 3(b). The data fit well to a model where the only source of decoherence is the expansion of the cloud along the $k$ vector of the microwaves, i.e., mechanism (ii). In free space, the velocity of each molecule along the $k$ vector is constant so the phase accumulated changes linearly with time. Consequently, this decoherence can be reversed by employing a spin-echo sequence in which a $\pi$ pulse is applied midway between the two $\pi / 2$ pulses. Figure 3(c) shows Ramsey fringes in free space with a $\pi$ pulse applied $14.7 \mathrm{~ms}$ after the first $\pi / 2$ pulse. Molecules at different positions experience different microwave powers, so a $\pi$ or $\pi / 2$ pulse cannot be perfect for all molecules. The extra $\pi$ pulse for the spin-echo technique thus causes an overall reduction in contrast. The signal also falls as the molecules expand and drop out of the detection region. However, we see no decoherence at all on this timescale; our data restrict the magnitude of any effect to less than $20 \mathrm{~s}^{-1}$ at the $95 \%$ confidence level.

The exceptionally small magnetic sensitivity of the transition $|1\rangle_{\text {str }} \leftrightarrow|2\rangle_{\text {str }}$ raises the question of whether the sensitivity might be even smaller in an excited vibrational state, $v>0$. To consider this, we expand the calculated $g_{l}$ and $g_{r}$ for $\mathrm{CaF}$ as a Taylor series in the bond length $R$ around the equilibrium value $R_{e}$. For a harmonic oscillator, only even-order terms in this expansion contribute to expectation values. For a slightly anharmonic oscillator such as most chemically bound molecules, both the linear and quadratic terms contribute significantly to the dependence on $v$. We calculate $g_{l}$ and $g_{r}$ at two additional bond lengths, close to the inner and outer turning points for the $v=1$ state of $\mathrm{CaF}$, to evaluate the coefficients linear and quadratic in $\left(R-R_{e}\right)$. Combining these with the expectation values of $\left(R-R_{e}\right)$ and $\left(R-R_{e}\right)^{2}$ for $\mathrm{CaF}$ yields

$$
\begin{aligned}
& g_{l}(v)=-1.86 \times 10^{-3}\left[1+6.20 \times 10^{-3}\left(v+\frac{1}{2}\right)+\ldots\right] \\
& g_{r}(v)=-5.13 \times 10^{-5}\left[1-5.77 \times 10^{-3}\left(v+\frac{1}{2}\right)+\ldots\right] \\
& g_{l}(v) / g_{r}(v)=36.16\left[1+0.0120\left(v+\frac{1}{2}\right)+\ldots\right]
\end{aligned}
$$


This indicates that the dependence on $v$ is small, and that the magnetic sensitivity is lowest for $v=0$. Nevertheless, the magnitude of the effect suggests that vibrational state dependence may be useful for fine-tuning magnetic sensitivity in other molecules.

In conclusion, we have shown that many laser-coolable molecules have pairs of states belonging to neighbouring rotational manifolds whose magnetic moments are large and identical to a few parts per million. In $\mathrm{CaF}$, the transition between the stretched states of $N=1$ and $N=2$ has a magnetic sensitivity of $-4.7(2) \mathrm{Hz} \mathrm{G}^{-1}$. We have demonstrated a coherence time of 6.4(8) ms for a magnetically trapped sample in a superposition of these states. Characterization of the principal sources of decoherence suggests that modifications to the trapping geometry and reduction of the cloud size will yield considerable improvements, with the prospect of coherence times greater than $1 \mathrm{~s}$ for small clouds at $5 \mu \mathrm{K}$ or below. These results open up potential new platforms for quantum simulation and computation with polar molecules in magnetic microtraps [22] and provide a route to the regime of strong coupling between solid-state systems and molecules held in magnetic chip traps [5]. These magnetically insensitive transitions may also be valuable in precision measurement [55], where sensitivity to magnetic fields is an important source of systematic error.

Underlying data may be accessed from Zenodo [56] and used under the Creative Commons CCZero license.

We thank J. Dyne and V. Gerulis for expert technical assistance. This work was supported by EPSRC under Grants No. EP/M027716/1 and No. EP/P01058X/1, and by the Royal Society. J. Aldegunde acknowledges funding by the Spanish Ministry of Science and Innovation (Grant No. PGC2018-096444-B-I00).

L. C. and H. J. W. contributed equally to this work.

[1] L. D. Carr, D. DeMille, R. V. Krems, and J. Ye, Cold and ultracold molecules: Science, technology and applications, New J. Phys. 11, 055049 (2009).

[2] R. Barnett, D. Petrov, M. Lukin, and E. Demler, Quantum Magnetism with Multicomponent Dipolar Molecules in an Optical Lattice, Phys. Rev. Lett. 96, 190401 (2006).

[3] H. P. Büchler, E. Demler, M. Lukin, A. Micheli, N. Prokof'ev, G. Pupillo, and P. Zoller, Strongly Correlated 2D Quantum Phases with Cold Polar Molecules: Controlling the Shape of the Interaction Potential, Phys. Rev. Lett. 98, 060404 (2007).

[4] A. Micheli, G. Pupillo, H. P. Büchler, and P. Zoller, Cold polar molecules in two-dimensional traps: Tailoring interactions with external fields for novel quantum phases, Phys. Rev. A 76, 043604 (2007).

[5] A. André, D. DeMille, J. M. Doyle, M. D. Lukin, S. E. Maxwell, P. Rabl, R. J. Schoelkopf, and P. Zoller, A coherent all-electrical interface between polar molecules and mesoscopic superconducting resonators, Nat. Phys. 2, 636 (2006).

[6] P. Rabl, D. DeMille, J. M. Doyle, M. D. Lukin, R. J. Schoelkopf, and P. Zoller, Hybrid Quantum Processors: Molecular Ensembles as Quantum Memory for Solid State Circuits, Phys. Rev. Lett. 97, 033003 (2006).

[7] D. DeMille, Quantum Computation with Trapped Polar Molecules, Phys. Rev. Lett. 88, 067901 (2002).

[8] D. Peter, S. Müller, S. Wessel, and H. P. Büchler, Anomalous Behavior of Spin Systems with Dipolar Interactions, Phys. Rev. Lett. 109, 025303 (2012).

[9] N. Y. Yao, A. V. Gorshkov, C. R. Laumann, A. M. Läuchli, J. Ye, and M. D. Lukin, Realizing Fractional Chern Insulators in Dipolar Spin Systems, Phys. Rev. Lett. 110, 185302 (2013).

[10] A. Micheli, G. K. Brennen, and P. Zoller, A toolbox for lattice-spin models with polar molecules, Nat. Phys. 2, 341 (2006).

[11] T. Karman and J. M. Hutson, Microwave Shielding of Ultracold Polar Molecules, Phys. Rev. Lett. 121, 163401 (2018).

[12] J. W. Park, Z.Z. Yan, H. Loh, S. A. Will, and M. W. Zwierlein, Second-scale nuclear spin coherence time of ultracold ${ }^{23} \mathrm{Na}^{40} \mathrm{~K}$ molecules, Science 357, 372 (2017).

[13] S. Kotochigova and D. DeMille, Electric-field-dependent dynamic polarizability and state-insensitive conditions for optical trapping of diatomic polar molecules, Phys. Rev. A 82, 063421 (2010).

[14] F. Seeßelberg, X.-Y. Luo, M. Li, R. Bause, S. Kotochigova, I. Bloch, and C. Gohle, Extending Rotational Coherence of Interacting Polar Molecules in a Spin-Decoupled Magic Trap, Phys. Rev. Lett. 121, 253401 (2018).

[15] S. S. Kondov, C.-H. Lee, K. H. Leung, C. Liedl, I. Majewska, R. Moszynski, and T. Zelevinsky, Molecular lattice clock with long vibrational coherence, Nat. Phys. 15, 1118 (2019).

[16] J. F. Barry, D. J. McCarron, E. B. Norrgard, M. H. Steinecker, and D. DeMille, Magneto-optical trapping of a diatomic molecule, Nature (London) 512, 286 (2014).

[17] H. J. Williams, S. Truppe, M. Hambach, L. Caldwell, N. J. Fitch, E. A. Hinds, B. E. Sauer, and M. R. Tarbutt, Characteristics of a magneto-optical trap of molecules, New J. Phys. 19, 113035 (2017).

[18] L. Anderegg, B. L. Augenbraun, E. Chae, B. Hemmerling, N. R. Hutzler, A. Ravi, Al. Collopy, J. Ye, W. Ketterle, and J. M. Doyle, Radio Frequency Magneto-Optical Trapping of CaF with High Density, Phys. Rev. Lett. 119, 103201 (2017).

[19] A. L. Collopy, S. Ding, Y. Wu, I. A. Finneran, L. Anderegg, B. L. Augenbraun, J. M. Doyle, and J. Ye, 3D MagnetoOptical Trap of Yttrium Monoxide, Phys. Rev. Lett. 121, 213201 (2018).

[20] D. J. McCarron, M. H. Steinecker, Y. Zhu, and D. DeMille, Magnetic Trapping of an Ultracold Gas of Polar Molecules, Phys. Rev. Lett. 121, 013202 (2018).

[21] H. J. Williams, L. Caldwell, N. J. Fitch, S. Truppe, J. Rodewald, E. A. Hinds, B. E. Sauer, and M. R. Tarbutt, Magnetic Trapping and Coherent Control of Laser-Cooled Molecules, Phys. Rev. Lett. 120, 163201 (2018).

[22] Y. Wang, T. Tran, P. Surendran, I. Herrera, A. Balcytis, D. Nissen, M. Albrecht, A. Sidorov, and P. Hannaford, Trapping ultracold atoms in a sub-micron-period triangular magnetic lattice, Phys. Rev. A 96, 013630 (2017). 
[23] J. Brown and A. Carrington, Rotational Spectroscopy of Diatomic Molecules (Cambridge University Press, Cambridge, 2003). See p. 351 for Zeeman Hamiltonian and p. 635 for fine and hyperfine Hamiltonian.

[24] I. C. Bowater, J. M. Brown, and A. Carrington, Microwave spectroscopy of nonlinear free radicals. I. General theory and application to the Zeeman effect in HCO, Proc. R. Soc. A 333, 265 (1973).

[25] F. Neese, The ORCA program system, Interdisc. Rev. Comput. Mol. Sci. 2, 73 (2012).

[26] P. J. Stephens, F. J. Devlin, C. F. Chabalowski, and M. J. Frisch, $A b$ initio calculation of vibrational absorption and circular dichroism spectra using density functional force fields, J. Phys. Chem. 98, 11623 (1994).

[27] P. Pollak and F. Weigend, Segmented contracted errorconsistent basis sets of double- and triple- $\zeta$ valence quality for one- and two-component relativistic all-electron calculations, J. Chem. Theory Comput. 13, 3696 (2017).

[28] E. van Lenthe, E. J. Baerends, and J. G. Snijders, Relativistic regular two-component Hamiltonians, J. Comput. Phys. 99, 4597 (1993).

[29] E. van Lenthe, E. J. Baerends, and J. G. Snijders, Relativistic total energy using regular approximations, J. Comput. Phys. 101, 9783 (1994).

[30] E. van Lenthe, E. J. Baerends, and J. G. Snijders, Geometry optimizations in the zero order regular approximation for relativistic effects, J. Comput. Phys. 110, 8943 (1999).

[31] K. Aidas, C. Angeli, K. L. Bak, V. Bakken, R. Bast, L. Boman, O. Christiansen, R. Cimiraglia, S. Coriani, P. Dahle, E. K. Dalskov, U. Ekström, and T. Enevoldsen, DALTON, a molecular electronic structure program, Release 2017.alpha, Wiley Interdisc Rev. Comput. Mol. Sci. 4, 269 (2014).

[32] See Supplemental Material at http://link.aps.org/supplemental/ 10.1103/PhysRevLett.124.063001 for further details of electronic structure calculations of $g_{r}$ and $g_{l}$, which includes Refs. [33-47].

[33] J. Aldegunde and J. M. Hutson, Hyperfine structure of ${ }^{2} \Sigma$ molecules containing alkaline-earth-metal atoms, Phys. Rev. A 97, 042505 (2018).

[34] R. F. Curl, The relationship between electron spin rotation coupling constants and $g$-tensor components, Mol. Phys. 9, 585 (1965).

[35] M. A. Anderson, M. D. Allen, and L. M. Ziurys, Millimeterwave spectroscopy of $\mathrm{MgF}$ : Structure and bonding in alkaline-earth monofluoride radicals, J. Chem. Phys. 100, 824 (1994).

[36] W. J. Childs, L. S. Goodman, and I. Renhorn, Radiofrequency optical double-resonance spectrum of SrF: The $X^{2} \Sigma^{+}$state, J. Mol. Spectrosc. 87, 522 (1981).

[37] L. B. Knight, Jr., J. M. Brom, Jr., and W. Weltner, Jr., Hyperfine interaction and chemical bonding in the $\mathrm{BeH}$ molecule, J. Chem. Phys. 56, 1152 (1972).

[38] P. F. Bernath, J. H. Black, and J. W. Brault, The spectrum of magnesium hydride, Astrophys. J. 298, 375 (1985).

[39] L.-E. Berg and L. Klynning, Rotational analysis of the A-X and B-X band systems of CaH, Phys. Scr. 10, 331 (1974).
[40] L. B. Knight, Jr. and W. Weltner, Jr., Hyperfine interaction and chemical bonding in $\mathrm{MgH}, \mathrm{CaH}, \mathrm{SrH}$, and $\mathrm{BaH}$ molecules, J. Chem. Phys. 54, 3875 (1971).

[41] J. M. Brom, Jr. and W. Weltner, Jr., ESR spectrum of the BeOH molecule, J. Chem. Phys. 64, 3894 (1976).

[42] J. M. Brom, Jr. and W. Weltner, Jr., ESR spectrum and structure of the $\mathrm{MgOH}$ radical, J. Chem. Phys. 58, 5322 (1973).

[43] L. M. Ziurys, W. L. Barclay, Jr., and M. A. Anderson, The millimeter-wave spectrum of the $\mathrm{CaOH}$ radical $\left(X^{2} \Sigma^{+}\right)$, Astrophys. J. 384, L63 (1992).

[44] M. A. Anderson, W. L. Barclay, and L. M. Ziurys, The millimeter-wave spectrum of the $\mathrm{SrOH}$ and SrOD radicals, Chem. Phys. Lett. 196, 166 (1992).

[45] W. Weltner, Jr., Magnetic Atoms and Molecules (Dover, New York, 1983), pp. 50-51.

[46] C. E. Mohn, D. J. D. Wilson, O. B. Lutnæs, T. Helgaker, and K. Ruud, The rotational $g$ tensor as a benchmark for $a b$ initio molecular property calculations, Adv. Quantum Chem. 50, 77 (2005).

[47] T. J. Lee and P. R. Taylor, A diagnostic for determining the quality of single-reference electron correlation methods, Int. J. Quantum Chem. 36, 199 (1989).

[48] S. Truppe, H. J. Williams, M. Hambach, L. Caldwell, N. J. Fitch, E. A. Hinds, B. E. Sauer, and M. R. Tarbutt, Molecules cooled below the Doppler limit, Nat. Phys. 13, 1173 (2017).

[49] J. A. Blackmore, L. Caldwell, P. D. Gregory, E. M. Bridge, R. Sawant, J. Aldegunde, J. Mur-Petit, D. Jaksch, J. M. Hutson, B. E. Sauer, M. R. Tarbutt, and S. L. Cornish, Ultracold molecules for quantum simulation: rotational coherences in $\mathrm{CaF}$ and RbCs, Quantum Sci. Technol. 4, 014010 (2018).

[50] W. J. Childs, G. L. Goodman, and L. S. Goodman, Precise determination of the $\nu$ and $N$ dependence of the spinrotation and hyperfine interactions in the $\mathrm{CaF} \mathrm{X}^{2} \Sigma_{1 / 2}$ ground state, J. Mol. Spectrosc. 86, 365 (1981).

[51] See for example Sec. 5.2 of M. R. Tarbutt, J. J. Hudson, B. E. Sauer, and E. A. Hinds, Prospects for measuring the electric dipole moment of the electron using electrically trapped polar molecules, Faraday Discuss. 142, 37 (2009).

[52] L. Caldwell, J. A. Devlin, H. J. Williams, N. J. Fitch, E. A. Hinds, B. E. Sauer, and M. R. Tarbutt, Deep Laser Cooling and Efficient Magnetic Compression of Molecules, Phys. Rev. Lett. 123, 033202 (2019).

[53] L. W. Cheuk, L. Anderegg, B. L. Augenbraun, Y. Bao, S. Burchesky, W. Ketterle, and J.M. Doyle, $\Lambda$-Enhanced Imaging of Molecules in an Optical Trap, Phys. Rev. Lett. 121, 083201 (2018).

[54] T. Bergeman, G. Erez, and H. J. Metcalf, Magnetostatic trapping fields for neutral atoms, Phys. Rev. A 35, 1535 (1987).

[55] See for example M. Kajita, Prospects of detecting $m_{e} / m_{p}$ variance using vibrational transition frequencies of ${ }^{2} \Sigma$-state molecules, Phys. Rev. A 77, 012511 (2008).

[56] https://doi.org/10.5281/zenodo.3382223. 